ANL/FPP/TM-135

16. 1693 $\frac{161}{9-8.86}$

\title{
GRILL: A CODE TO CALCULATE THE SPECTRUM OF LOWER-HYBRID WAVES LAUNCHED BY A BRAMBILLA WAVEGUIDE ARRAY
} by C. D. Boley

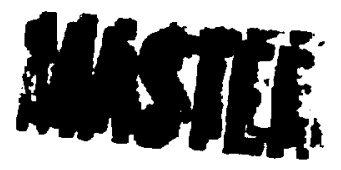

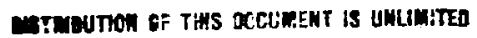

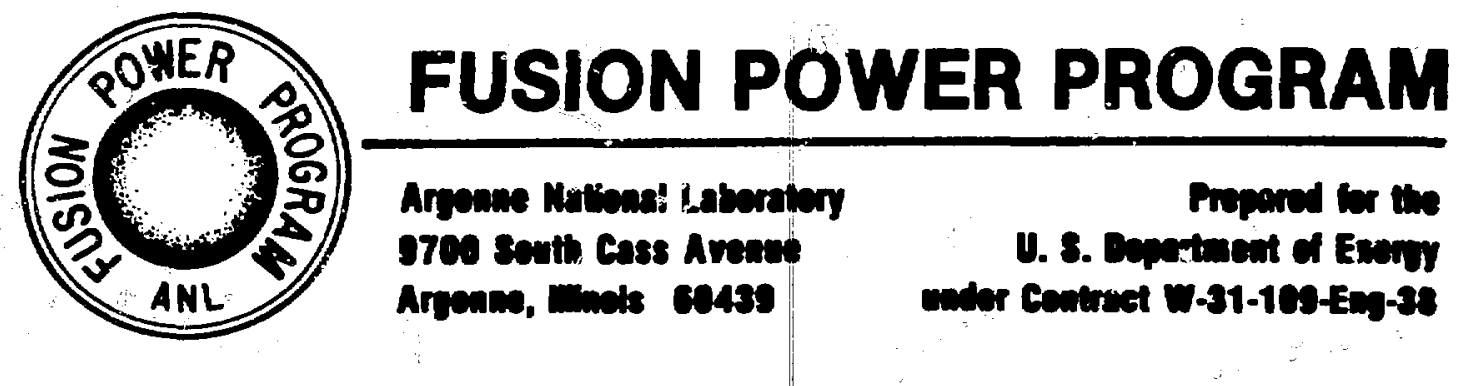


Distribution Categories:

Magnetic Fusion Energy (UC-20)

Plasma Systems (UC-20a)

Fusion Systems (UC-20d)

ANL/ FPP/TM-135

ARGONNE NATIONAL LABORATORY

9760 South Cass Avenue

Argonne, Illinois 60439

GRILL: A CODE TO CALCULATE THE SPECTRUM OF LOWER-HYBRID WAVES

LAUNCHED BY A BRAMBILLA WAVEGUIDE ARRAY

by

C. D. Boley

Fusion Power Program

\section{June 1980}

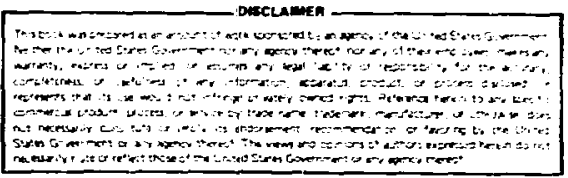

$\checkmark$ 


\section{TABLE OF CONTENTS}

Page

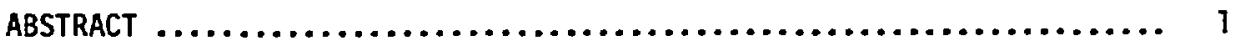

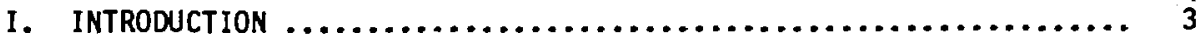

II. SUMMARY OF THE BRAMBILLA THEORY $\ldots \ldots \ldots \ldots \ldots \ldots \ldots \ldots \ldots \ldots \ldots \ldots$

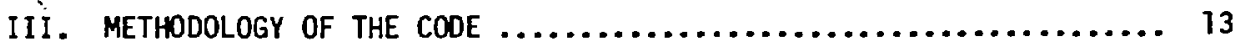

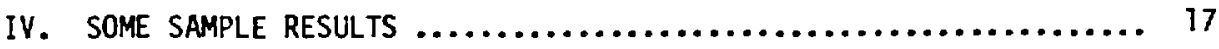

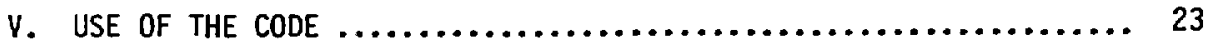

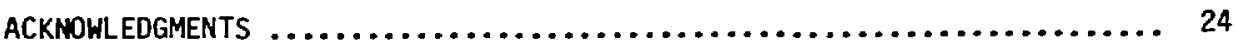

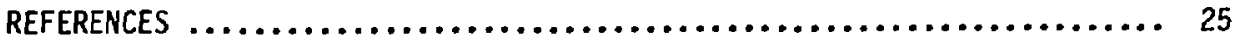

LIST OF FIGURES

No.

Title $\quad$ Page

1 Geometry of the waveguide array $\ldots \ldots \ldots \ldots \ldots \ldots \ldots \ldots \ldots \ldots . \ldots . \ldots . \ldots$

2 Central peak in the spectrum of the electric field at the cutoff distance $x_{c}$, for waveguides consisting of $N$ ports $(N=6,12,24$, 36). All other parameters, including the phase difference $\Delta \phi$ across each port, are held fixed $\ldots \ldots \ldots \ldots \ldots \ldots \ldots \ldots \ldots \ldots \ldots, 18$

3 Average reflection coefficient $\vec{R}$ and fractional intensity $\varepsilon\left(1.82 k_{0}, 1.20 k_{0} ; x_{c}\right)$ as a function of the vacuun distance. Waveguide array as in Fig. 2, with $N=12$

4 Average reflection coefficient and fractional intensity as a function of the electron density gradient. Same waveguide array and interval of $k_{z}$ as in the preceding figure .................. 
GRILL: A COOE TO CALCULATE THE SPECTRUM OF LOWER-HYBRID WAVES LAUNCHEO BY A BRAMBILLA WAVEGUIDE ARRAY

\author{
C. D. Boley \\ Fusion Power Program
}

\begin{abstract}
A computer code for calculating the predictions of the Brambilla theory of lower-hybrid wave launching is documented.
\end{abstract}




\section{INTRODUCTION}

The STARFIRE design calls for the use of lower hybrid waves for plasma heating and current drive $[1,2]$. The excitation of these waves via a phased multi-waveguide structure has been discussed by Brambilla [3], whose theory gives the fields up to a distance at which wave damping can no longer be ignored. The subsequent Landau damping of the waves and the implications for heating and current drive have been investigated by Fisch [4], and the inplications for steady-stats reactors such as STARFIRE have been explored by Ehst [5].

Brambilla wrote a computer code for his theory, but it has not been publicly documented and made available to the fusion community. Since the results are important for the STARFIRE design, and since the theory is not prohibitively complicated, the decision was made to write an independent code. This document describes that code, called GRILL. The code has been designed for maximum flexibility, in that, for example, it can handle any number of waveguide ports, with variable widths and spacings.

The original article by Brambilla does not explicitiy give all of the significant results (for example, the fields in the plasma), and there are some unfortunate misprints. For these reasons, it was thought useful to begin with a summary of the basic equations of the theory (Section II). This section also draws on the exposition by Krapchev and Bers [6]. Section III describes the general strategy involved in the numerical treatment of the equations. Some sample results and trends are discussed in Section IV. Finally, the operation of the code itself is described in Section $V$. 


\section{SUMMAPY OF THE BRAMBILLA THEORY}

The waveguide structure is illustrated in Fig. 1. As this figure indicates, the waveguides open into the wall defined by the $y-z$ plane, with the $x$ axis pointing toward the plasma. The toroidal field (assumed to be straight) is parallel to the $z$ axis. Along this direction there is a series of $N$ ports, with the edges of the $p$-th port located at $z=z_{p}$ and $z=z_{p}+b_{p}$ (thus the width is $\left.b_{p}\right)$. The ports are separated by a series of perfectly conducting slabs, the width of the slab separating ports $p$ and $p+1$ being $d_{p}=z_{p+1}-\left(z_{p}+b_{p}\right)$. The ports are taken to extend indefinitely in the y direction.

Within the waveguides $(x \leq 0)$, the fields are taken to be, in MKS units,

$$
\begin{aligned}
E_{z}(x, z)= & E_{0} \sum_{p=1}^{N} \theta_{p}(z) \sum_{n=0}^{M}\left(\delta_{n 0} \alpha_{p} e^{i k_{n p} x}+B_{n p} e^{-i k_{n p} x}\right) \\
& \times \cos \frac{n \pi\left(z-z_{p}\right)}{b_{p}}, \\
B_{y}(x, z)= & -\frac{E_{0}}{c} \sum_{p=1}^{n} \theta_{p}(z) \sum_{n=0}^{M} \frac{k_{0}}{k_{n p}}\left(\delta_{n 0} p_{p} e^{i k_{n p} x}-\beta_{n p} e^{-i k_{n p} x}\right) \\
& x \cos \frac{n \pi(z-z)}{b_{p}}, \\
E_{x}(n, z)= & -\frac{i c}{k_{0}} \frac{\partial B_{y}(x, z)}{\partial z},
\end{aligned}
$$




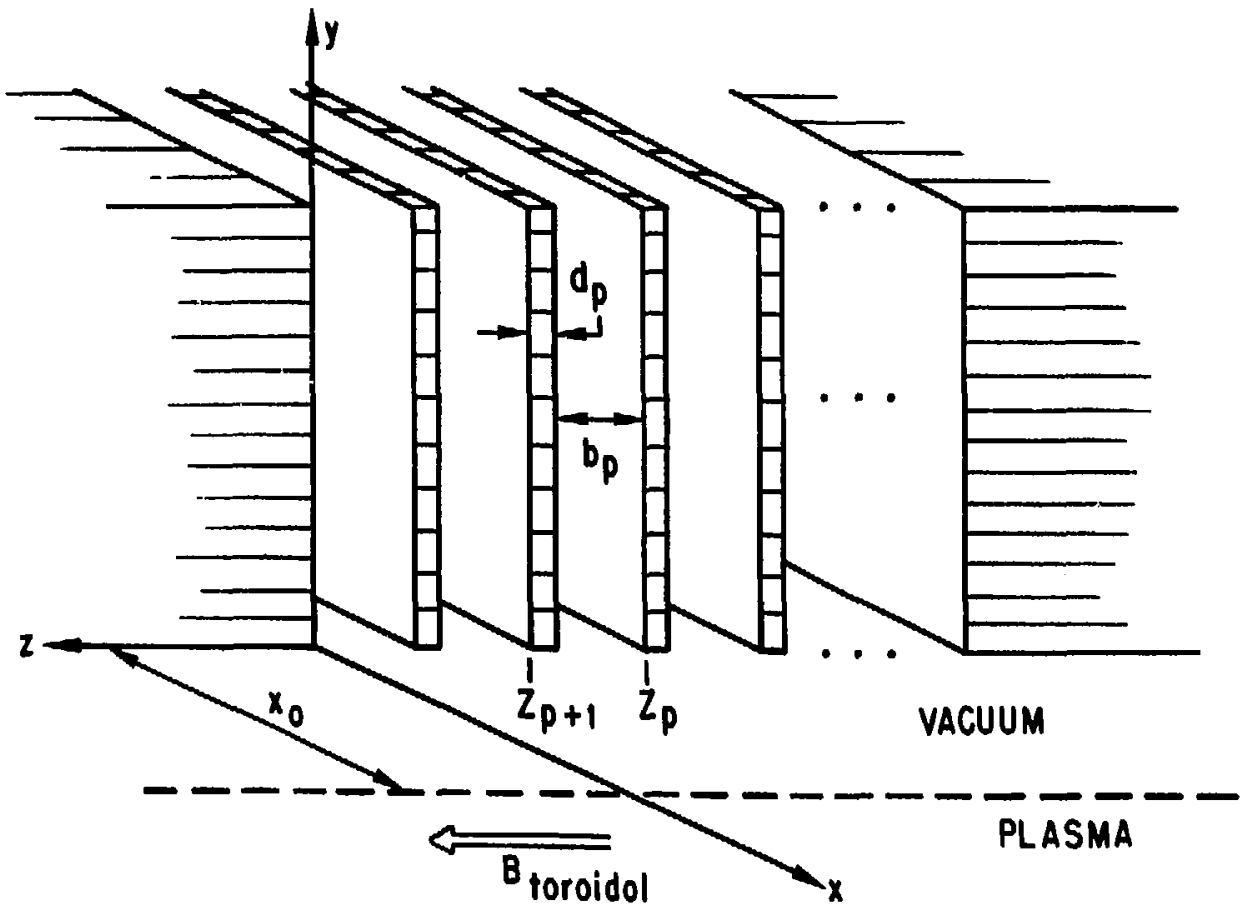

Fig. 1. Geometry of the waveguide array. 
where $\theta_{p}(z)$ is unity for $z$ within the $p$-th waveguide $\left(z_{p} \leq z \leq z_{p}+b_{p}\right)$ and zero e1sewhere. The $n$-sums extend over propagating and evanescent modes. The frequency $\omega_{0}$ and the waveguide widths are assumed here to satisfy $\pi / b_{p}>\omega_{0} / c$, so that there is only a single propagating mode $n=0$ in each waveguide (the modifications to accommodate more propagating modes are trivial). The wave vectors are then given by

$$
\begin{aligned}
& k_{\text {op }}=\frac{\omega_{0}}{c}=k_{0} \text {, } \\
& k_{n p}=i\left[\left(n \pi / b_{p}\right)^{2}-k_{0}^{2}\right]^{\frac{2}{2}}, \quad n \geq 1
\end{aligned}
$$

The number of evanescent modes is, in principle, infinite but is truncated to $M$ for computational practicality. The dimensionless complex excitation coefficients $\alpha_{p}$ are input parameters; the complex coefficients $B_{n p}$ for the reflected wave and for the evanescent modes are to be determined. The constant $E_{0}$ is an overall multiplicative factor. Note that the magnetic field is purely transverse to the waveguide axis $x$, while the electric field has both transverse and longitudinal components.

Solving for the time-averaged Poynting vector in the $x$-direction, one sees that the total power delivered by the waveguide array to the plasma is

$$
P=\frac{E_{0}^{2}}{2 \mu_{0} c} \sum_{p} b_{p}\left[\left|\alpha_{p}\right|^{2}-\left|\beta_{0 P}\right|^{2}\right] \text {, }
$$

per unit length in the $y$ direction.

Outside the waveguides, there is a vacuum region of width $x_{0}$ (possibly zero) followed by the plasma region. In the vacuum $\left(0 \leq x \leq x_{0}\right)$ the fielós are written as 


$$
\begin{aligned}
& E_{z}(x, z)=\dot{E}_{0} \int_{-\infty}^{\infty} d k_{z} e^{i k_{z} z} \sigma\left(k_{z}\right)\left[e^{i k_{x} x}-Y\left(k_{z}\right) e^{-i k_{\dot{x}} x}\right], \\
& B_{y}(x, z)=-\frac{E_{0}}{c} \int_{-\infty}^{\infty} d k_{z} \frac{k_{0}}{k_{x}} e^{i k_{z} z} \sigma\left(k_{z}\right)\left[e^{i k_{x} x}+Y\left(k_{z}\right) e^{-i k_{x} x}\right] \\
& E_{x}(x, z)=-\frac{i c}{k_{0}} \frac{\partial B_{y}(x, z)}{\partial z},
\end{aligned}
$$

where

$$
k_{x}= \begin{cases}\left(k_{0}^{2}-k_{z}^{2}\right)^{\frac{3}{2}}, & \left|k_{z}\right|<k_{0} \\ i\left(k_{z}^{2}-k_{0}^{2}\right)^{\frac{3}{2}}, & \left|k_{z}\right|>k_{0}\end{cases}
$$

The Fourier coefficients $\sigma\left(k_{z}\right)$ and $Y\left(k_{z}\right)$ are as yet unknown. If there is no plasma, Y vanishes.

Within the plasma $\left(x \geq x_{0}\right)$, the fields are represented as

$$
\left(\begin{array}{l}
E_{z}(x, z) \\
E_{x}(x, z) \\
B_{y}(x, z)
\end{array}\right)=E_{0} \int_{-\infty}^{\infty} d k_{z} e^{i k_{z} z}\left(\begin{array}{c}
\mathscr{E}_{z}\left(x, k_{z}\right) \\
E_{x}\left(x, k_{z}\right) \\
\beta_{y}\left(x, k_{z}\right)
\end{array}\right),
$$

and the Fourier amplitudes on the right-hand side satisfy equations which depend on the particular plasma model chosen. In the model for lower hybrid waves used by Brambilla and by Krapcher and Bers, these equations are

$$
\frac{\partial^{2} \mathscr{E}_{z}}{\partial x^{2}}+\left(k_{0}^{2}-k_{z}^{2}\right)\left(1-\frac{x-x_{p}}{L}\right) \mathscr{E}_{z}=0,
$$




$$
i k_{z} \frac{\partial E_{z}}{\partial x}-\left(k_{0}^{2}-k_{z}^{2}\right) E_{x}=0
$$

The magnetic field follows from Faraday's law:

$$
i \omega_{0} \beta_{y}=i k_{z} \mathscr{E}_{x}-\frac{\partial \mathscr{E}_{z}}{\partial x}
$$

In Eq. (7a) a constant electron density gradient has been assumed, and $L=$ $\varepsilon_{0} m_{e} \omega_{0}^{2} /\left(e^{2} d n_{e} / d x\right)$ is the cutoff length into the plasma, at which the electron plasma frequency equals the driving frequency. The assumption of a constant density gradient is an obvious idealization, and it might besprofitable to rework the theory with, say, a density which increases expontially with the distance from the wall. The solution for $\varepsilon_{z}$ in terms of Airy functions, used below, would no longer hold, but the basic formalism would remain intact.

Returning to Eq. (7a), there are two kinds of solutions, depending on the sign of $k_{0}^{2}-k_{z}^{2}$. Defining

$$
\begin{aligned}
a\left(k_{z}\right) & =L^{2 / 3}\left|k_{0}^{2}-k_{z}^{2}\right|^{1 / 3}, \\
\bar{x} & =\frac{x-x_{0}}{L},
\end{aligned}
$$

one has, for $\left|k_{z}\right|<k_{0}$,

$$
\epsilon_{z}\left(x, k_{z}\right)=c_{0}\left(k_{z}\right) A i[a(\bar{x}-1)],
$$

and for $\left|k_{z}\right|>k_{0}$,

$$
E_{z}\left(x, k_{z}\right)=c_{i} \cdot\left(k_{z}\right)\{\operatorname{Ai}[a(1-\bar{x})]+i B i[a(1-\bar{x})]\},
$$


where $A i$ and $B i$ are Airy functions. The functions $c_{0}\left(k_{z}\right)$ and $c_{1}\left(k_{z}\right)$ are as yet undetermined.

Now the fields are to be matched at the interfaces between the various regions. At the waveguide-vacuum interface, $E_{z}$ is continuous for all $z$, and $B_{y}$ is continuous for values of $z$ corresponding to the waveguide openings. These requirements lead to the following system of linear equations relating the $\beta$ 's to the $\alpha^{\prime} s$ :

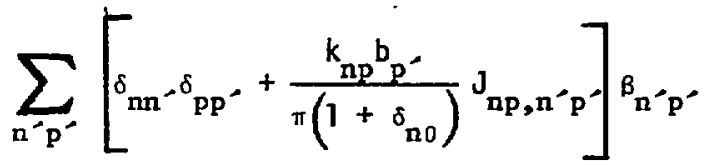

$$
\begin{aligned}
& =\sum_{p^{\prime}}\left[\delta_{n 0^{\prime} \delta^{\prime}}-\frac{k_{n p^{\prime}}^{b} p^{\prime}}{\pi\left(1+\delta_{n 0}\right)} J_{n p^{\prime}, p^{-}}\right] \alpha_{p^{\prime}},
\end{aligned}
$$

where $\mathrm{J}_{\mathrm{np}, \mathrm{n}^{\prime} \mathrm{p}^{-}}$is the dimensionless integral

$$
\begin{aligned}
& J_{n, p, n^{\prime} p^{-}}=\frac{1}{b_{p^{b} p^{-}}} \int_{-\infty}^{\infty} d k_{z} \frac{1+y\left(k_{z}\right)}{1-y\left(k_{z}\right)} e^{i k_{z}\left(z_{p^{-z}} z^{-}\right)} \frac{k_{z}^{2}}{k_{x}} \\
& \times \frac{1-(-1)^{n} e^{i k_{z} b_{p}}}{k_{z}^{2}-\left(n \pi / b_{p}\right)^{2}} \frac{1-(-1)^{n^{-}} e^{-i k_{z} b_{p^{\prime}}}}{k_{z}^{2}-\left(n^{-} \pi / b_{p^{\prime}}\right)^{2}} .
\end{aligned}
$$

This integral contains the function $Y\left(k_{z}\right)$, which is determined when the fields are matched at the vacuum-plasma interface. The matching calculations result in

$$
Y\left(k_{z}\right)=n\left(k_{z}\right) \frac{1-Z\left(k_{z}\right)}{1+Z\left(k_{z}\right)} \text {, }
$$


where, for $\left|k_{z}\right|<k_{0}$,

$$
\begin{aligned}
& \eta\left(k_{z}\right)=\exp \left[2 i x_{0}\left(k_{\sigma}^{2}-k^{2}\right)^{\frac{7}{2}}\right], \\
& Z\left(k_{z}\right)=i a^{\frac{1}{2}} \frac{A i(-a)}{A i+(-a)},
\end{aligned}
$$

and for $\left|k_{z}\right|>k_{0}$,

$$
\begin{aligned}
& n\left(k_{z}\right)=\exp \left[-2 x_{0}\left(k_{z}^{2}-k_{0}^{2}\right)^{\frac{1}{2}}\right] ; \\
& Z\left(k_{z}\right):=a^{\frac{3}{2}} \frac{A i(a)+i B i(a)}{A i-(a)+i B i-(a)} .
\end{aligned}
$$

With the information in Eqs. (12)-(15), Eq. (11) is a closed linear system for the reflection parameters $\beta_{n p}$. After this system is solved, via the code described below, the fields can be obtained. The electric field amplitudes [cf. Eqs. (10)] are then

$$
\left(\begin{array}{l}
c_{0}\left(k_{z}\right) \\
c_{1}\left(k_{z}\right)
\end{array}\right)=\frac{T\left(k_{z}\right)}{2 \pi} n^{-\frac{2}{2}} \frac{\eta-\gamma}{1-\gamma}\left(\begin{array}{l}
{[A i(-a)]^{-1}} \\
{[A i(a)+i B i(a)]^{-1}}
\end{array}\right),
$$

where

$$
T\left(k_{z}\right)=-i k_{z} \sum_{n p}\left(\delta_{n j} \alpha_{p}+\beta_{n p}\right) \frac{1-(-1)^{n} e^{-i k_{z} b_{p}}}{k_{z}^{2}-\left(n \pi / k_{p}\right)^{2}} .
$$

of course the electric field is continuous at $k_{z}=k_{0}$. The $k_{z}$ spectrum of the square of the electric field, $I\left(x, k_{z}\right)$, which is defined by 


$$
\int_{-\infty}^{\infty} d z\left|E_{z}(x, z)\right|^{2}=\int_{-\infty}^{\infty} \frac{d k_{z}}{2 \pi} I\left(x, k_{z}\right),
$$

is then (for $x$ in the plasma)

$$
I\left(x, k_{z}\right)=\left|T n^{-\frac{3}{2}} \frac{n-Y}{1-Y} g(\bar{x})\right|^{2},
$$

where

$$
g(\bar{x})=\left\{\begin{array}{ll}
\frac{\operatorname{Ai}[a(\bar{x}-1)]}{A i(-a)} & \left|k_{z}\right|<k_{0} \\
\frac{A i[a(1-\bar{x})]+i B i[a(1-\bar{x})]}{A i(a)+i B i(a)} & \left|k_{z}\right|>k_{0}
\end{array} .\right.
$$

Finally we note the power spectrum $P\left(x, k_{z}\right)$, which is the integrand on the right-hand side of

$$
\int_{-\infty}^{\infty} d z S_{x}(x, z)=\int_{-\infty}^{\infty} \frac{d k_{z}}{2 \pi} P\left(x, k_{z}\right),
$$

where $\vec{S}$ is the Poynting vector. Outside the waveguides, the power spectrum is independent of position and works out to be

$$
P\left(k_{z}\right)=\left\{\begin{array}{ll}
0 & \left|k_{z}\right|<k_{0} \\
\frac{k_{0} E_{0}^{2}}{\mu_{0} c} \frac{|T|^{2}}{\left(k_{z}^{2}-k_{0}^{2}\right)^{\frac{7}{2}}} \frac{\operatorname{Im}(Y)}{|1-Y|^{2}} & \left|k_{z}\right|>k_{0}
\end{array},\right.
$$

The spectrum is, of course, nonnegative, and its integral gives the total power $P$ of Eq. (3). 


\section{METHODOLOGY OF THE CODE}

The code divides logically into two parts. In the first part the matrix

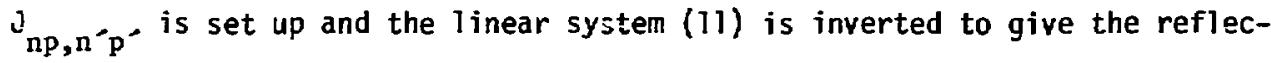
tion parameters $\beta_{n p}$. In the second part the spectra are evaluated. These parts are now described in turn.

In evaluating $\mathrm{J}_{\mathrm{np}, \mathrm{n}^{-} \mathrm{p}^{-}}$, one notes first that the integrand contains resonant denominators which can vanish. These zeros are canceled by zeros in the numerator. In addition, the factor $k_{x}$ in the denominator introduces an integrable singularity at $k_{z}= \pm k_{0}$. To avoid numerical pathologies, therefore, one introduces the transformation

$$
k_{z}=\left\{\begin{array}{ll}
k_{0} \cos \theta & \left|k_{z}\right|<k_{0} \\
k_{0} \cosh u & \left|k_{z}\right|>k_{0}
\end{array} .\right.
$$

One then has

$$
\begin{aligned}
J_{n p, n^{\circ} p^{-}=} & \int_{0}^{\frac{2}{2} \pi} d \theta \frac{1+Y}{1-Y} f_{\left.n p, n^{-p}-\left[\zeta_{0}(\theta)\right]\right]} \\
& -i \int_{0}^{\infty} d u \frac{1+Y}{1-Y} f_{n p, n^{\prime} p}-\left[\zeta_{1}(u)\right],
\end{aligned}
$$

where 


$$
\begin{aligned}
& \zeta_{0}(\theta)=b_{\mathrm{pp}}-k_{0} \cos \theta, \\
& \zeta_{1}(u)=b_{\mathrm{pp}}-k_{0} \cosh u,
\end{aligned}
$$

and

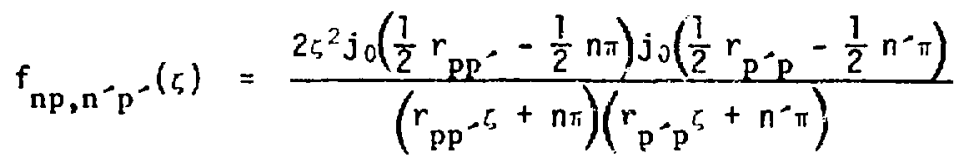

$$
\begin{aligned}
& \times \cos \left[\frac{5}{b_{p p^{-}}}\left(z_{p}-z_{p^{\prime}}\right)+\frac{1}{2}\left(r_{p p^{-5}}-n \pi\right)-\frac{1}{2}\left(r_{p^{-p} p^{5}-n^{-} \pi}\right)\right],
\end{aligned}
$$

and also

$$
b_{p^{\prime}}=\left(b_{p^{\prime}} b_{p^{\prime}}\right)^{\frac{1}{2}}, \quad r_{p^{\prime}}=\left(\frac{b_{p}}{b_{p^{\prime}}}\right)^{\frac{2}{2}} .
$$

Written in this form, the integral can be handled by standard numerical techniques. In the code it is evaluated by the routine ANC4 [7], which employs a 9-point Newton-Cotes formula over subintervals, returning an answer with an accuracy specified by a parameter EP in the calling sequence. It was found to be adequate to take EP equal to $5 \times 10^{-6}$, which gives an accuracy of five or six figures.

The symmetry property $J_{n p, n^{-} p^{-}}=J_{n^{\prime} p^{\prime}, n p}$ is utilized to reduce the number of integrations. If the waveguide array is uniform (all widths equal and all separations equal), then $J_{n p, n^{-}}$- depends on the waveguide indices only via the combination $p-p^{-}$. The code checks whether the array is uniform and, if it is, takes advantage of this additional simplification.

The Airy functions implicit in $\mathrm{J}_{\mathrm{np}, \mathrm{n}^{-} \mathrm{p}^{-}}$are evaluated by taking the first ten terms of their Taylor series expansions. This approximation, which is an 
expansion about an infinitely sharp density gradient, was more than adequate in all cases considered. The approximation of taking only the first term [6] usually gave reflection ccefficients accurate to within 15 or $20 \%$, but sometimes the results were off by a factor of two.

Finally, the matrix equation (11) is solved. This is a complex inear system in $N \times(M+1)$ unknowns, where $M$ is the number of evanescent modes accounted for. The accuracy of the final spectra increases rapidly as a function of $M$, and for accuracy to three or four figures it was found sufficient to take $M=2$. The actual inversion of Eq. (11) is accomplished by calling the subroutine LEQTIC in the IMSL Library [8].

In the second part of the code, which concerns the evaluation of the spectra, two regions of $k_{z}$ are considered. First the spectra are evaluated in the "central region", $0 \leq k_{z} \leq 3 k_{0}$ (with a spacing of $0.02 k_{0}$ ), the details of which are important for current drive studies. In this region it is very useful to know the fraction of the total electric field spectrum which lies between any two wave vectors:

$$
\varepsilon\left(k_{z}, k_{z}^{\prime} ; x\right)=\int_{k_{z}^{\prime}}^{k_{z}} d \bar{k}_{z} I\left(x, \bar{k}_{z}\right)\left[\int_{-\infty}^{\infty} d \bar{k}_{z} I\left(x, \bar{k}_{z}\right)\right]^{-1},
$$

To facilitate the calculation of this quantity, the code calculates the fraction of the total area lying below each $k_{z}$ in the region. Numerically, this is taken to be

$$
\varepsilon\left(k_{z},-\infty ; x\right) \simeq \int_{-60 k_{0}}^{k_{2}} d \bar{k}_{z} I\left(x, \bar{k}_{z}\right)\left[\int_{-60 k_{0}}^{60 k_{0}} d \bar{k}_{z} I\left(x, \bar{k}_{z}\right)\right]^{-1},
$$

and the same integration routine as before is used. The desired fraction can then be read off from the output as 


$$
\varepsilon\left(k_{z}, k_{z}^{\prime} ; x\right)=\varepsilon\left(k_{z},-\infty ; x\right)-\varepsilon\left(k_{z}^{\prime},-\infty ; x\right)
$$

After printing out these results, the code evaluates the spectra in the broader region defined by $-60 k_{0} \leq k_{z} \leq 60 k_{0}$ (spacing of $0.2 k_{0}$ ), which is of interest for heating studies.

The sole numerical subtlety in this part of the code is the treatment of the function $T\left(k_{z}\right)$, Eq. (17). Like the quantity $J_{n p, n * p}$. discussed earlier, this function has resonant denominators and canceling zeros in the numerator. Rewritten so as to be free of numerical proolems, it is, for $k_{z} \neq 0$,

$$
\begin{aligned}
T\left(k_{z}\right)= & \sum_{n p}\left(\delta_{n q}^{\alpha}+k_{n p}\right) \frac{b_{p}^{2} k_{z}}{b_{p} k_{z}+s n \pi} \exp \left\{-i\left[k_{z} z_{p}+\frac{1}{2}\left(k_{z} b_{p}-s n \pi\right)\right]\right\} \\
& \times j_{0}\left[\frac{1}{2}\left(b_{p} k_{z}-s n \pi\right)\right],
\end{aligned}
$$

where $s$ is the sign of $k_{z}$. The value at the origin is

$$
T(0)=\sum_{p} b_{p}\left(\alpha_{p}+\beta_{o p}\right) .
$$




\section{SOME SAMPLE RESULTS}

The Brambilla theory has two types of input parameters: those associated with the waveguides (the number of ports, the widths, the separations, and the driving amplitudes, as well as the frequency), and those associated with the plasma model (the vacuum distance and the density gradient). This is a very large parameter space. In order to develop an understanding for some of the results, we now briefly consider the behavior as the number of ports, the vacuum distance, and the density gradient are varied, with the other parameters held fixed. These last parameters are assigned values consistent with the preliminary STARFIRE design, namely:

$$
\begin{aligned}
& \nu \equiv \omega_{0} / 2 \pi=1.4 \mathrm{GHz} \\
& \left.\begin{array}{l}
b_{p}=4.0 \mathrm{~cm} \\
d_{p}=0.7 \mathrm{~cm}
\end{array}\right\} \text { (uniform -ill) } \\
& a_{\mathrm{p}}=\exp (2 \pi i \mathrm{p} / 3) \text { (uniform driving amplitudes; } 3 \text { ports per wavelength) }
\end{aligned}
$$

First we consider the characteristics of the central peak in the spectrum of the electric field, as a function of the number $N$ of waveguides. For sufficiently .large $N$, this peak is expected to occur at a value of $k_{z}$ such that $2 \pi / k_{z}$ equals the grill width associated with a phase difference of $2 \pi$, or $3\left(b_{p}+d_{p}\right)$; thus $k_{z} / k_{0}=1.52$. This is borne out by Fig. 2 , which shows the central peak for $N=6,12,24$, and 36 , at fixed values of $x_{0}$ and $d n_{e} / d x$ and with $x$ at the cutoff distance into the plasma. Another important feature is the fact that the product of the width and the number of ports is approximately constant. This is a diffraction effect, since the trend remains when the plasma 


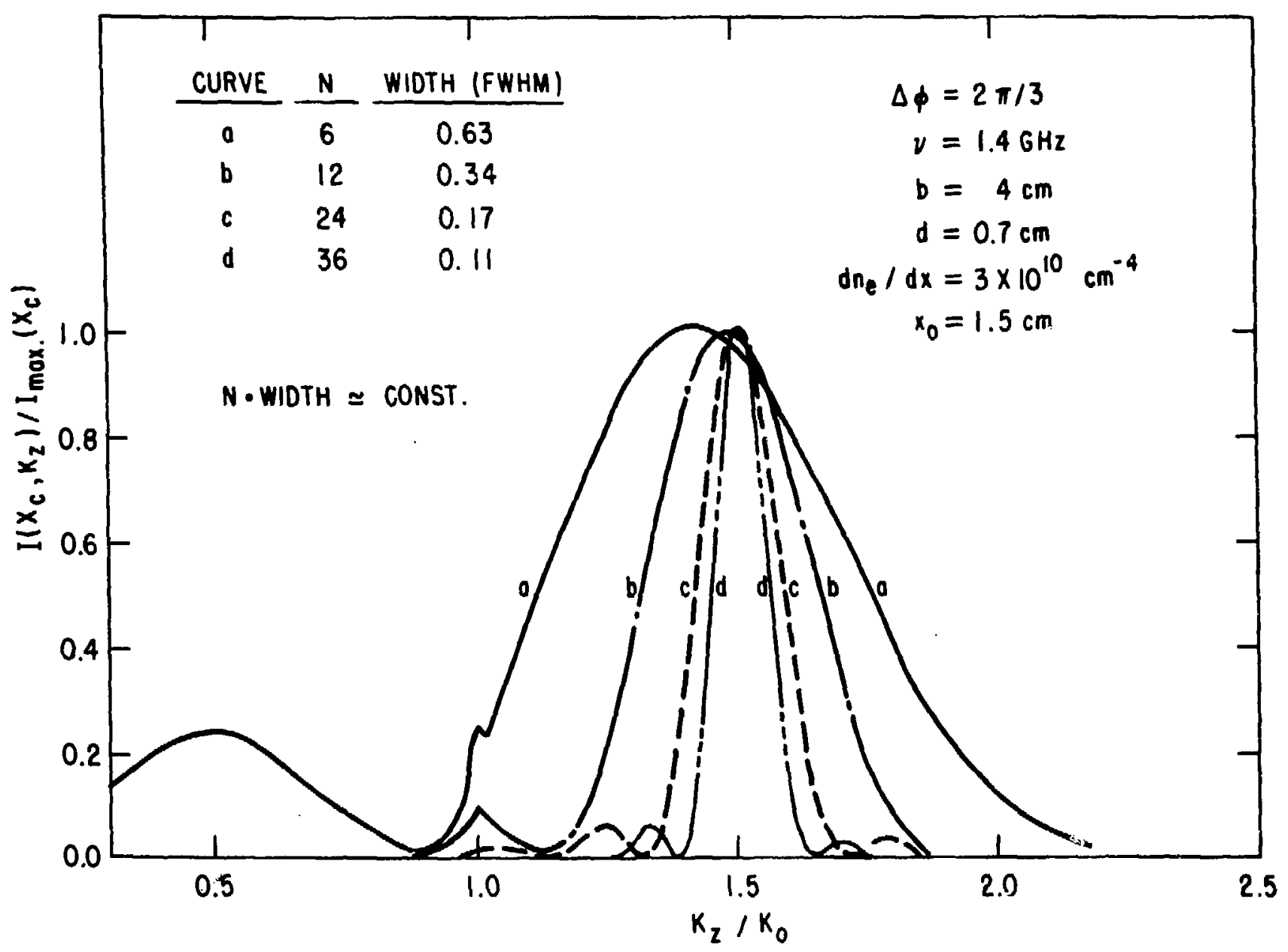

Fig. 2. Central peak in the spectrum of the electric field at the cutoff distance $x_{c}$ for waveguides consisting of $N$ ports $(N=6,12,24,36)$. All other parameters, including the phase difference $\Delta \phi$ across each port, are held fixed. 
parameters are varied, or when the plasma is removed (the value of the product., however, varies from case to case). Note also the peak at $k_{z}=k_{0}$, corresponding to wave propagating with the free-space wavelength, which disappears as $N$ is increased. For any given range of $k_{z}$, plots like Fig. 2 allow for an estimate of the minimum number of ports necessary to concentrate the spectrum in that range.

Next we hold the number of ports fixed at $N=12$ and vary the vacuum distance $x_{0}$, with the density gradient. having the same value as in the previous case. The significant output parameters to be noted now are the average reflection coefficient $\bar{R}$, defined by

$$
\bar{R}=\sum_{p} b_{p}\left|\beta_{o p}\right|^{2} / \sum_{p} b_{p}\left|\alpha_{p}\right|^{2}=\frac{1}{12} \sum_{p=1}^{12}\left|\beta_{o p}\right|^{2},
$$

and the fractional intensity $\varepsilon$ lying within a given range of $k_{z}$, which we take to be $1.20 k_{0} \leq k_{z} \leq 1.82 k_{0}$ (typical for STARFIRE). It is desired to minimize $\bar{R}$, which by definition is less than unity and to maximize $\varepsilon$. As Fig. 3 indicates, however, a tradeoff is involved in identifying the optimal vacuum distance. The reflection coefficient always increases with $x[3,6]$, but $\varepsilon$ also increases with $x_{0}$, at least until the reflection coefficient becomes prohibitely large. The reason for the trend in $E$ is that modes with $\left|k_{z}\right|>k_{0}$ tend to be damped out as they cross the vacuum, and the damping increases with $\left|k_{2}\right|$; thus modes with large $\left|k_{z}\right|$, and therefore far from the desired interval, become proportionallv less represented as $x_{0}$ increases.

Finally, we consider the behavior of $\bar{R}$ and $\varepsilon$ as the electron density gradient is varied, keeping $N=12$ and working with a representative vacuum distance of $1.5 \mathrm{~cm}$. The results are shown in Fig. 4, which illustrates that a tradeoff again is involved. Brambilla has given detailed arguments concerning the existence of an optimal density gradient for the reflection coefficient. It is 


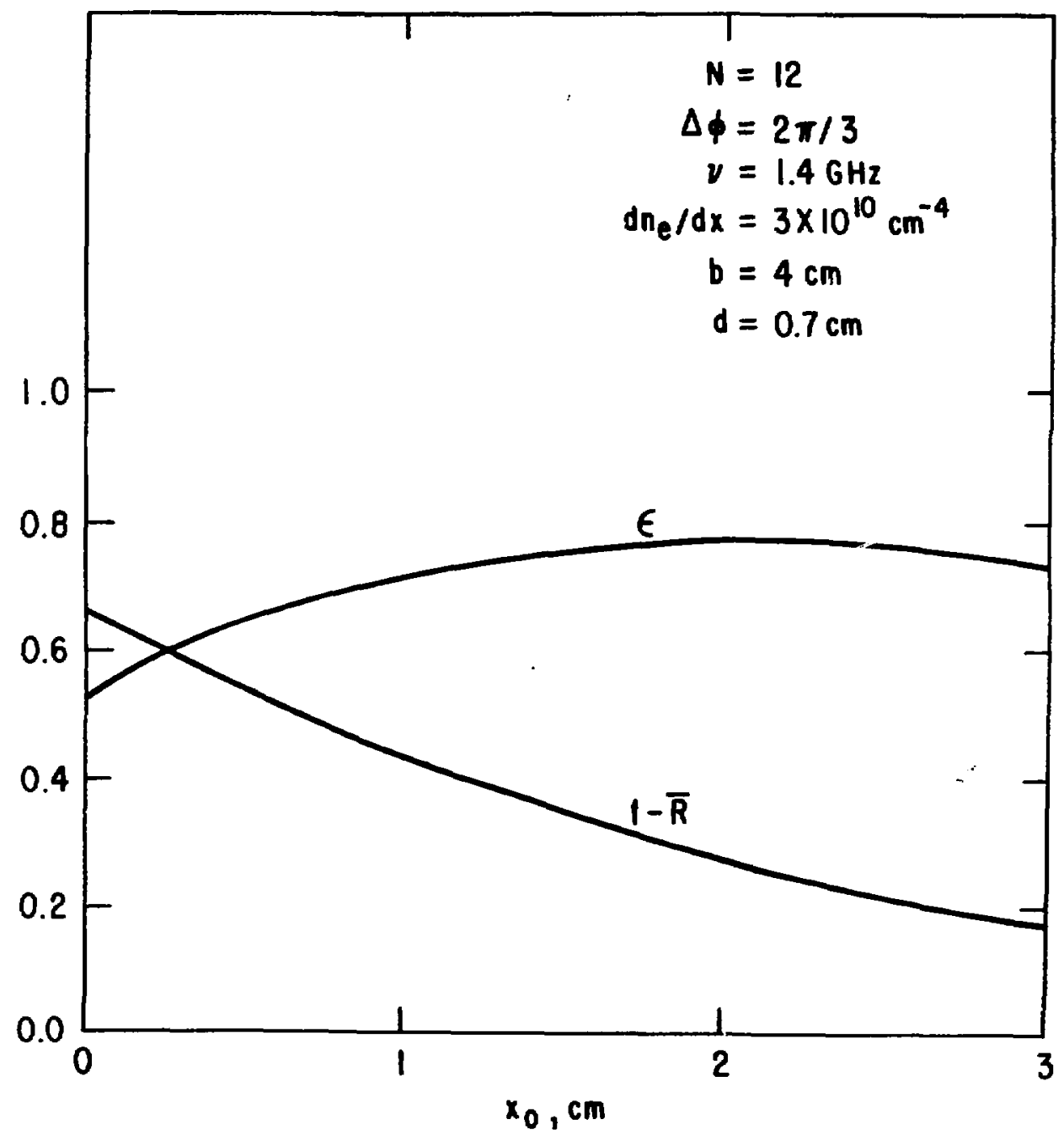

Fig. 3. Average reflection coefficient $\bar{R}$ and fractional intensity $\varepsilon\left(1.82 \mathrm{~kg}, 1.20 \mathrm{k}_{0} ; x_{c}\right)$ as a function of the vacuum distance. Waveguide array as in Fig. 2, with $N=12$. 


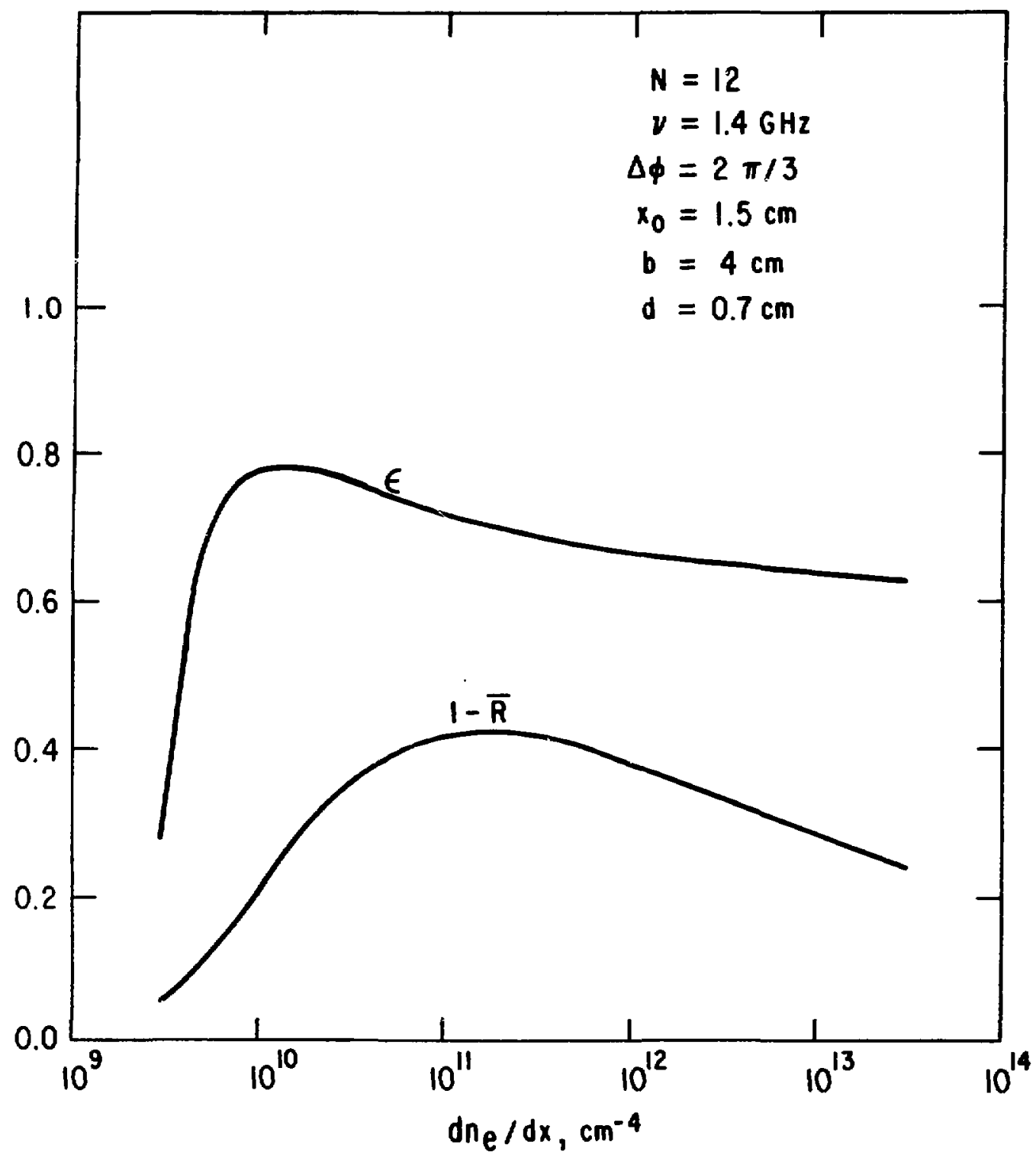

Fig. 4. Average reflection coefficient and fractional intensity as a function of the electron density gradient. Same waveguide array and interval of $k_{2}$ as in the preceding figure. 
fortunate that $\varepsilon$ varies rather slowly from its maximum value in the region where $1-\bar{R}$ peaks, so that a simultaneous optimization is possible.

Further results of the theory, and the methods of optimization, will be presented in the context of the STARFIRE design. 
The input to the code consists of the following parameters:

FREGHZ: $\quad \omega_{0} / 2 \pi$ in $\mathrm{GHz}$

EO: $\quad E_{0}$ in volts/cm

NWGUID: Number of waveguides

WIOTH(I): Widths $b_{p}$ of the ports in $\mathrm{cm}$

$\left.\begin{array}{ll}\text { ALPHA(I): } & \text { Amplitudes }\left|\alpha_{p}\right| \\ \text { PHI(I): } & \text { Phases of } \alpha_{p}, \text { divided by } 2 \pi\end{array}\right\} I=1, \ldots$ NWGUID

DIST(I): Distances between ports in $\mathrm{cm}, \mathrm{I}=1$, ... NWGUID-1

DXPL: $\quad x_{0}$ in $\mathrm{cm}$

DNDX: $\quad d n_{e} / d x$ in $\mathrm{cm}^{-4}$

NX: $\quad \begin{aligned} & \text { Number of positions } x_{j} \text { at which spectra are to be } \\ & \text { evaluated }\end{aligned}$

$\operatorname{XBAR}(J): \quad$ Values of $\left(x_{j}-x_{0}\right) / L, J=1, \ldots, N X$

NEVMOD: Number of evanescent modes to be included

IAIRY: Normally equal to unity. If zero, the Airy functions in $Z$ are approximated by their value at the origin, as is done in Ref. 6.

IPLASM: Normally equal to unity. If zero, no plasma is present. In this case DXPL and DNDX are not used, and the spectra are evaluated at a distance XBAR $\mathrm{cm}$ into the vacuum.

The arrays are dimensioned so that NWGUID $\leq 24$ and NEVMOD $\leq 3$, but these limits are ivially changed. Similarly $N X<10$ is assumed.

The order in which the input is read in is:

1. NWGUID, NEVMOD, NX, IAIRY, IPLASM

2. FREGHZ, EO, DXPL, DNDX

2(F10.6,E13.5)

3. $\operatorname{WIDTH}(I)$

(7F10.6)

4. $\operatorname{DIST}(I)$

(7F10.6) 
5. ALPHA(I)

6. $\mathrm{PHI}(\mathrm{I})$

7. $\operatorname{XBAR}(\mathrm{J})$
(7F 10.6)

(7F10.6)

(7F 70.6)

The output of the code is self-explanatory.

The code consists of about 500 FORTRAN cards and, with its present dimension statements, occupies $430 \mathrm{~K}$. A typical running time on the IBM $370 / 195$, for 13 waveguides and 2 evanescent modes, is $90 \mathrm{~s}$.

\section{ACKNOWLEDGMENTS}

The author is indebted to C. C. Baker and D. A. Ehst for useful comments and criticism. 


\section{REFERENCES}

[1] "STARFIRE, A Commercial Tokamak Reactor - An Interim Report," ANL/FPP/TM-125 (1979).

[2] C. C. Baker, et al., "STARFIRE, A Commercial Tokamak Power Plant Design," Nucl. Engr. Design (submitted).

[3] M. Brambilla, Nucl. Fusion 16, 47 (1976).

[4] N. J. Fisch, Phys. Rev. Letters 41, 873 (1978).

[5] D. A. Ehst, Nuel. Fusion 19, 1369 (1979).

[6] V. Krapchev and A. Bers, Nucz. Fusion 18, 519 (1978).

[7] Argonne National Latoratory, Applied Mathematics Division, ANL D158S.

[8] International Mathematical and Statistical Libraries, Library 1, Edition 6 (1977). 